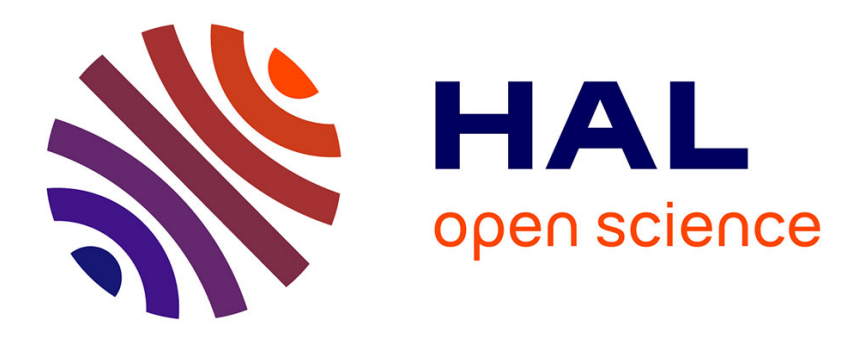

\title{
Remote Laboratory for Game-Based Distance Learning in Electronics
}

\author{
Franck Luthon, Benoît Larroque
}

\section{To cite this version:}

Franck Luthon, Benoît Larroque. Remote Laboratory for Game-Based Distance Learning in Electronics. 4th Int. Conference on Electronics, Communications and Networks (CECNet 2014), Dec 2014, Beijing, China. hal-01103183

\section{HAL Id: hal-01103183 \\ https://hal.science/hal-01103183}

Submitted on 14 Jan 2015

HAL is a multi-disciplinary open access archive for the deposit and dissemination of scientific research documents, whether they are published or not. The documents may come from teaching and research institutions in France or abroad, or from public or private research centers.
L'archive ouverte pluridisciplinaire HAL, est destinée au dépôt et à la diffusion de documents scientifiques de niveau recherche, publiés ou non, émanant des établissements d'enseignement et de recherche français ou étrangers, des laboratoires publics ou privés. 


\title{
Remote Laboratory for Game-Based Distance Learning in Electronics
}

\author{
Franck Luthon \& Benoît Larroque \\ University of Pau and Adour Province (UPPA) \\ Department of Industrial Engineering, Bayonne Institute of Technology (IUT), Anglet, France \\ Corresponding author: Franck.Luthon@univ-pau.fr
}

\begin{abstract}
The advances in communication networks and web technologies, in conjunction with the improved connectivity of test and measurement devices make it possible to implement e-learning applications that encompass the whole learning process. In electrical engineering, it means not only lecture, tutorial, quiz and simulation, but also practical labwork for training with real-world devices that are controlled remotely. To make e-labs attractive, they should be easily implemented and accessed on the web by a client. This keypoint raises technical issues that are discussed in this paper. Nonetheless pedagogical issues are equally important. Here, to foster student motivation, a game-like scenario embedded in a learning management system is proposed.
\end{abstract}

\section{INTRODUCTION}

This paper presents LaboREM, a remote laboratory implemented for e-learning in electronics. Bayonne Technological University Institute in Anglet, France, promotes the introduction of distance learning for the education of students in electrical and mechanical engineering, either to learn teleworking or for lifelong learning. As part of the academic curriculum for the bachelor of science degree in industrial engineering, the course of analog electronics deals with operational amplifiers (OAs), active filters and oscillators. The learning objectives are to implement basic electronic functions with OAs, and to use measurement devices for the characterization of circuits. The practical labwork assigned to students can be done remotely, instead of classical hands-on sessions lasting 3 to 4 hours each. Here, we focus on 1st year undergraduate students (teenagers of 18-19 years old). For that target audience who often lacks personal motivation in studying, both game-based learning and adaptive learning are good solutions in order to enhance students' motivation and autonomy.

The lab platform is based on the design and control of virtual instruments (VIs) for the management of remote experimentation through the web, together with the use of a Learning Management System (LMS) for the management of students and for the collaborative work supervision. The client-server architecture that allows remote control of instruments in realtime, with the management of both the stack of experiments and the list of users, is a classical solution.The originality of the proposed work is twofold: (i) a feel- ing of the lab via the use of a video camera and a robotic arm for placement of components, plus the optional use of computer vision to address user-specific requests to see the equipment in detail (zoom-in for displaying instruments or device under test DUT); this is intended to mimic both the user eye and the user hand; (ii) a game-based scenario (treasure hunt) that is implemented as learning approach, with an attempt to mimic the behaviour of students when they work close together (collaborative work through use of chat, forum) and with a learning itinerary adapted to each student (difficulty level depending on the evaluated knowledge and motivation).

The paper is organized as follows. In section 2, the motivations of the work are given with respect to the state of the art. Section 3 shows the technical choices for software architecture and hardware setup of the lab, while section 4 describes the game-like pedagogical scenario implemented via an LMS.

\section{MOTIVATIONS AND STATE OF THE ART}

Practice is known to be an essential part of learning in engineering education, as long as clear learning objectives are assigned (ABET criteria). The pros and cons of the three types of labs (hands-on, virtual and remote) are wellknown. Virtual labs are based on simulations only (mathematical models), whereas remote labs use real-world instruments in a physical lab (Garcia-Zubia \& Alves 2011). Virtual and remote labs belong to the generic group of e-labs, made possible thanks to the development of Internet. They are the modern counterpart of traditional hands-on 
labs. Since access to real-world labs is restricted due to high number of students, cost of equipment, supply and maintenance needs, e-labs are great alternatives. The complementarity of activities is also well known: a mixed pedagogy including hands-on, simulations and remote control is preferable, which is called blended learning. A specific argument in favor of real remote labs (compared to virtual labs) is the fact that nowadays in industry, more and more engineering activities take place remotely, for example the maintenance of systems or control of processes through a computer interface located in a distant supervision room (SCADA: supervisory control and data acquisition). Hence, there is a strong need to educate students to this new way of working.

However, the bottlenecks of remote labs lie in the interaction with instruments and the interaction between people. Smart software is needed in order to: (i) design an open (flexible enough) architecture for distant control of hardware devices (technical need) and (ii) develop tools and interfaces to enable and track the cognitive behavior of the user in a distant instrumentation application (human need). When distance activities are developed, motivational features are very important for the self-regulation of learning (Mendez \& Gonzalez 2011). To get real-time knowledge of the state of work of a student and for automatic assessment procedures, the use of an LMS is mandatory. Some authors already addressed the coupling of remote labs with LMS (Orduña et al. 2013), or the coupling of virtual labs with games, but the present work is one of the first attempts to mix three views together: remote lab, LMS and game. The lab is piloted by a web-based client application (accessed via a browser). Thanks to the LMS, the collaborative dimension is added to the remote labwork, together with the supervision of students activity. Moreover, interaction tools for better immersion and motivation are proposed: a Top 10 score is displayed in real-time to motivate students by a challenge, and a robotic arm plays the role of the student hand for choosing and placing components to build the DUT. This solution is an alternative to the virtual wiring of a relay switching matrix implemented in (Tawfik et al. 2013).

\section{ARCHITECTURE OF LABOREM}

\subsection{Software Architecture}

The software should enable a completely connectible application and easy installation by the client, run in real-time, communicate easily with hardware devices and with clients, enable extensibility (to add new experiments), implement collaborative work. The choice of network technology should therefore be compliant with various issues: accessibility, reusability, portability, security, scalability, interoperability, traceability. The importance of universality versus software power discussed in (Garcia-Zubia et al.
2009) leads to the conclusion that a service-oriented architecture (SOA) should be adopted. In (Guimaraes et al. 2011), a formal reference model for weblabs described in UML language identifies the major functions that a remote lab with SOA must support in order to yield the proper quality of service. Four main entities interact: (i) the client that uses the lab, (ii) the lab that operates the hardware resources for experiments, (iii) the LMS that organizes the learning activity, and (iv) the corporate webserver that gives access to the platform. LaboREM is compliant with this model (Fig. 1). Based on the use of two servers,

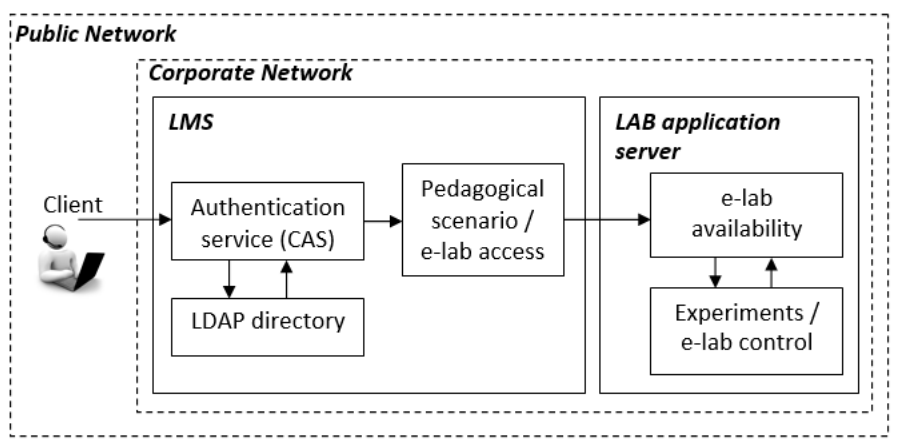

Figure 1. Network and software architecture.

it is designed to make the LMS communicate with the remote lab. The LMS is the middleware including databases for management of users (using lightweight directory access protocol LDAP), central authentication service (CAS), administration, learning tools, content packaging, evaluation, communication tools for collaborative work. The remote lab is the hardware part that includes instruments (with software drivers). The labwork is accessed through the use of the LMS, by calling, at specific steps in the learning sequence, the proper URL address corresponding to the remote VI to pilot. The e-lab availability service is taken in charge by the labserver itself (management of the queue of users to give access to experiments). The e-lab control service is for piloting the remote devices (instruments, robot). Compared to other existing solutions like NetLab (Nedic \& Machotka 2007) or iLab shared architecture (Harward et al. 2008), the main differences are as follows: the collaborative tools are provided by the LMS, not by the remote lab. A single labserver is in use, and most of the services are taken in charge by the LMS (authentication, trace recording, data storage), apart from the actual task of interaction with hardware.

\subsection{Hardware architecture}

In addition to the corporate webserver, the lab application server located inside the industrial engineering department laboratory is another dedicated server for connection to the lab equipment (Fig. 2). A typical hardware setup consists of instruments connected through GPIB (waveform generator, multimeter, oscilloscope), plus other equipment connected through 


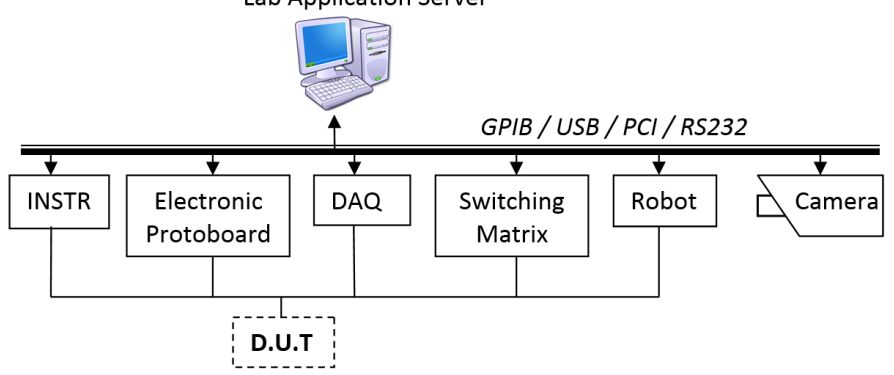

Figure 2. Hardware architecture: INSTR means instruments; DAQ means data acquisition.

USB, RS232, PCI or wireless (data acquisition board, electronic protoboard, switching matrix with relays, on-off button, robotic arm, camera, automatic background lighting).

Robotic Arm for Component Placement: LaboREM gives the opportunity to build the circuit using an old fashioned robotic arm (Fig. 3). The DUTs are circuits consisting of OAs, resistors and capacitors only (no inductance). Thus, a simple solution for easily placing components is to equip them with magnets, so that the right place and good connection contact are achieved, without needing high spatial precision or big mechanical effort. Fig. 3 shows the two-step action: first, grasp one component from the bank of components; then, move and drop it down on the receiver board connected to the breadboard where OAs are already in place. In addition to the robot-built

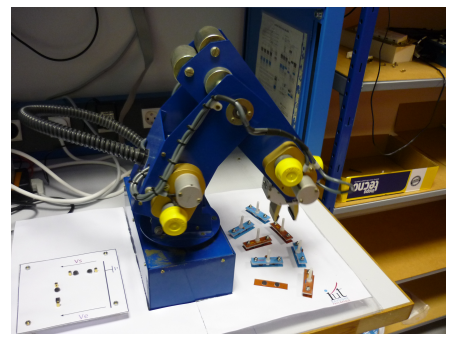

(a)

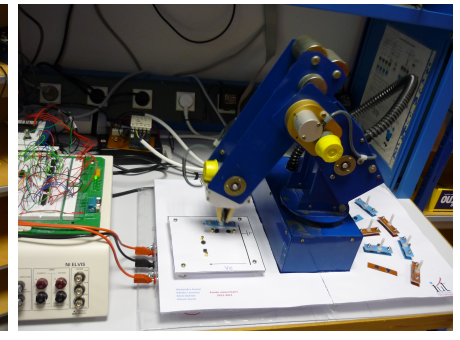

(b)

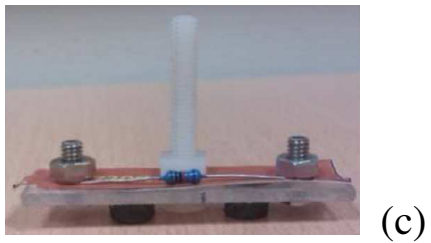

Figure 3. Robot in action for (a) choosing and (b) placing a component equipped with magnets (c).

filters, there are 8 precabled passive and active filters on the breadboard, so that the student can choose either to build his own filter, or to use precabled ones among high-pass, low-pass, band-pass, rejection, plus one extra unknown filter to be uncovered. Choosing the DUT is done in the client interface by selecting the type of filter and component values (components can be chosen among 8 values: 4 resistors, 4 capacitors). Note that in case of failure of the robot placement, the client can switch to precabled filters instead, that do not require the robot use.

\subsection{Server-Side Application}

The block-diagram of the lab application is shown in Fig. 4. The application is made of four main VIs

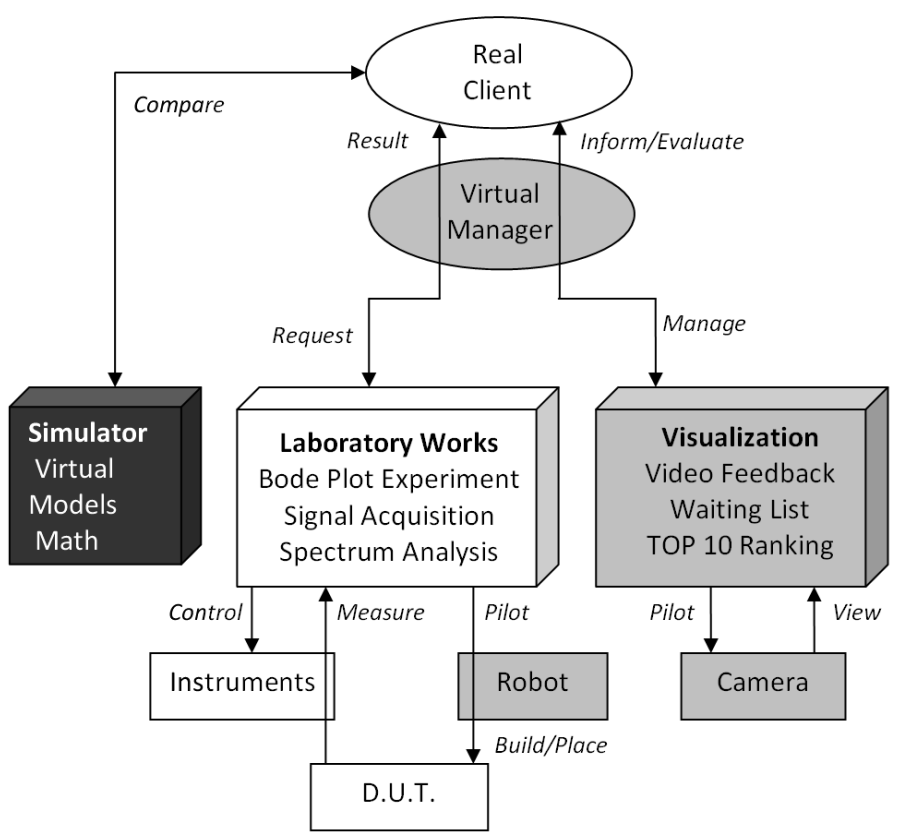

Figure 4. Application block-diagram: blocks in gray correspond to functions that are added to get a remote lab, starting from a hands-on lab (blocks in white).

listed below, that are designed and controlled with NILabVIEW software.

LaboratoryWorks is the main VI of the remote lab. It enables to select the type of experiment (GPIBBode plots or DAQ-Signal spectrum analyses) and type of filter. It allows to control the instruments and the robotic arm to build the DUT, to make measurements, to recover data graphs and to participate to Top 10 ranking. The client has full control over this VI.

Visualization gives a feedback on the remote lab. The client can observe this VI. It includes three functions: (i) VideoFeedback is for video capture and image processing in real-time. A template matching algorithm, applied on color and shape information in each image captured at video-rate, enables the user to focus selectively and zoom on each of the devices. (ii) WaitingList displays the waiting queue of users that are requesting the control on instruments. (iii) TOP10 displays the ranking in a "hall of fame" (best answers to measurement requests).

Simulator is directly accessible via the LMS. It is the main VI of the virtual lab (as opposed to the remote lab) that offers numerical simulations of the various DUTs. It gives students the opportunity to make comparisons between simulation results and actual measures. Since it does not communicate with any instrument, there is no waiting queue to manage: many users can run simulations in the same time.

Virtual Manager is in charge of the lab management. It ensures 3 main functions. (i) Storage: it stores data of each experiment (username, timer, experiment selection, Top 10 answers); (ii) Scheduling: it manages the waiting queue of connected clients. It uses a 
specific tool (Remote Panel Connection Manager) for the management of remote front panel connections. There is no sophisticated scheduling mechanism nor concurrency problem, since a user is allowed $5 \mathrm{~min}$ utes only, and a first-in first-out solution is adopted (no time slot reservation). (iii) Initialization and Security: it reinitializes the system (interface and robot) to its default state, each time an experiment ends, or if another user takes the control of the lab.

\subsection{Client-Side Communication Interface}

CGI scripts: A first solution is low-level programming with http protocol: to use CGI scripts (common gateway interface) to transmit information (Put, Get and Post instructions). It implies upload and download of data (input parameters and output measurements). The deployment on client-side is a desktop application: executable installed and running on the client computer (Steriu \& Luthon 2006). The main drawback is that it only works in batch mode (asynchronous communication), so that real-time interaction is not possible Another drawback is that the desktop application must be updated by the client each time the lab-server application undergoes a software upgrade.

Remote Front Panels: A 2nd solution is to use remote front panels (RFP) available with NI-LabVIEW software. Starting from an existing VI including drivers to communicate locally with instruments, the web publishing tool, with remote control option activated, allows to create a HTML file embedding the front panel object code, so that a VI is visible and controllable from Internet in real-time. As soon as the lab application server (LabVIEW webserver) is started, the HTML file is accessible over the Internet through its specific URL address. Therefore it is both easy for a client to use the lab and for a tutor to add a new lab activity: there is very little programming overhead needed to publish new VIs on the web. The front panel seen by the remote client is exactly the same as the one seen within the local application: the interface is rich and convivial (Fig. 5). The main drawback is that RFP client-server data communication uses a proprietary non-http protocol. It might by detected and stopped by some firewalls, anti-viruses or proxies. A second drawback is that the client has to download and install the shareware LabVIEW RunTime Engine (LVRTE) which is a plugin of size 40Mbytes. Fortunately, since the latest release of LabVIEW 2014, most browsers support this plugin: not only Internet Explorer, but also Chrome, Firefox, Netscape, Safari or Opera. A final point worth mentioning is that the VI is executed on the server-side. Clients who are connected to the remote front panel, and waiting to get the control over the VI, can observe what the current client who has the control is doing. Note that this can be a good point for student collaboration.

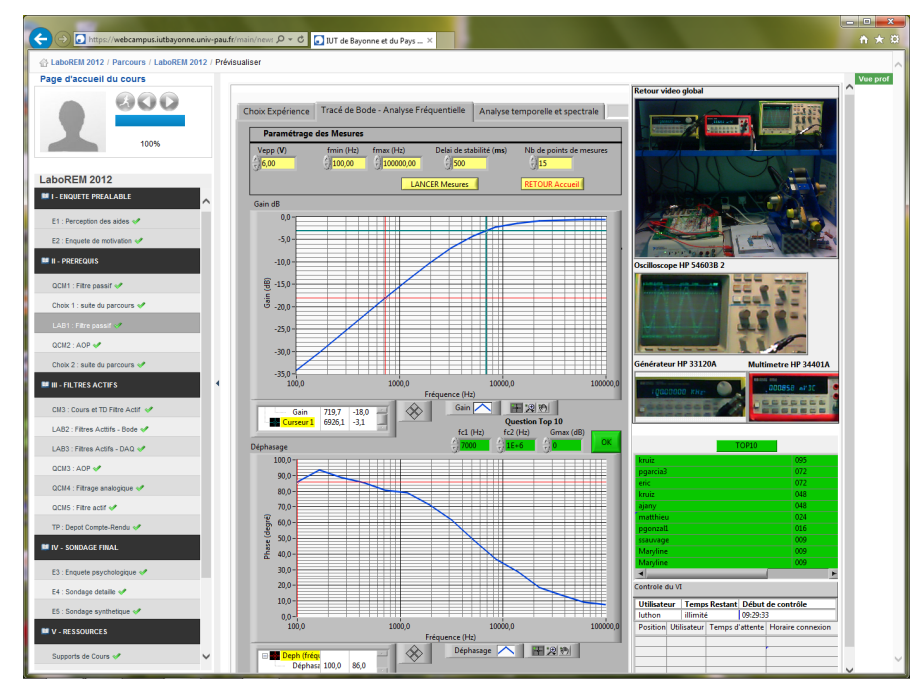

Figure 5. Client rich interface showing a remote front panel: left) learning steps of the pedagogical scenario; center) experimental results (Bode plots of a high-pass filter); right) video feedback of lab and instruments, Top 10 list and queuing list of users.

Webservices: A third solution is the use of webservices (Dutta et al. 2011). The advantage is to conform to standard communication protocol (fully compatible with any network and web browser configuration, no restriction due to firewall or antiviruses, mobile device compatibility). The client interface may be thin. A specific URL address with command parameters generates dataflows in real-time, containing output data described in XML, TEXT or JSON format. This solution may even be deployed starting from a LabVIEW project (that provides a tool to build Restful Webservices and a WebUI Builder). This alternative yields a remote interface to LabVIEW that avoids the use of the non-http RFP protocol. Its main drawback is that the remote graphical interface requires dedicated programming. Adding a new lab activity is not straightforward since it requires extra coding first.

We have tested the three solutions for LaboREM, and we advocate the 3rd solution using webservices. The prospective use of HTML5, merging JavaScript and XML, is the best technical choice. Among others, it allows to address the flexibility and ubiquitous use of the application on mobile devices (smart phone, tablet) which is desirable for gaming purpose. Nevertheless, as the 2nd solution based on RFPs does not require extra-coding and provides a richer interface, we present here the results based on RFP: Fig. 5 shows a typical interface seen by a client (remote student) when asking for plotting Bode curves to characterize the transfer function of active filters. The user builds his own circuit by piloting the robotic arm, makes measurements in real-time, exploits graphs thanks to cursors and markers, optionally takes part in the Top 10 , has a visual feedback of the lab through a camera, and gets information about the waiting list of users with a time-counter, so that he knows exactly when he will be served. In the meanwhile, he can switch to other activities proposed in the pedagogical scenario. 


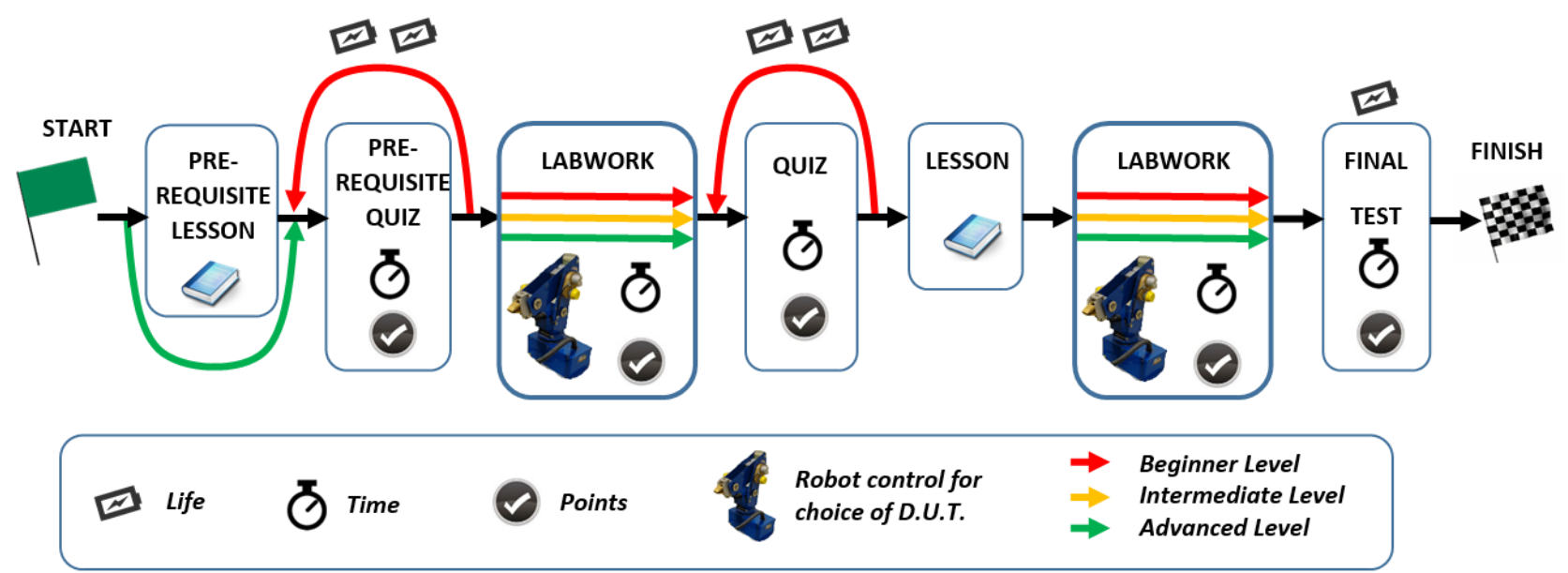

Figure 6. Learning map as a treasure hunt with various steps implemented as activities in the LMS: lessons (documentation and help), quizzes (self evaluation and final test), remote labworks.

\section{PEDAGOGICAL SCENARIO}

\subsection{Game-like strategy}

Motivation of students practicing e-learning may not only be modeled and measured, but also fostered, with the proper use of an LMS. In addition, the use of games may contribute in enhancing motivation. Games are characterized by 6 key-dimensions: fantasy, clear rules and goals, sensory stimuli, challenge, mystery and control. They are taken into account as follows: mystery (unknown filter to uncover), sensory stimuli (visual feedback and robotic motion), challenge (Top 10), control (choice of DUT), rules (limited time and number of trials), goals (clear learning objectives with three difficulty levels to choose from), fantasy (freedom to conceive one's own circuit). The student has the choice between various filters: some of them are known (SallenKey, Wien etc.) or can be built by the student himself; others are unknown and should be identified during the lab session. Depending on the filters chosen for study, and on the exactness of answers, the student accumulates more or less points during his "travel" and may take place in the "hall of fame". If he discovers what the unknown filter is, he has reached the final objective of the "quest". If he fails, he may choose to backtrack and try again (limited repetitions). All the scenario is managed by the LMS. The game-like scenario conceived to boost student motivation (Luthon et al. 2009) is based on 4 concepts as in e-games: levels, lives, points, and time. Four counters are used for evaluating student's activity: a timer, a counter for highest level achieved (difficulty level), a counter for number of lives used (number of trials), a counter for scoring (marks obtained to online quizzes and final exam). This allows to automatically estimate a participation index and a performance index which are taken into account as in (Mendez \& Gonzalez 2011) for driving motivation in auto-regulated distance e-learning. A polylinear learning path is implemented in the LMS as a "treasure hunt", that is adapted to the competence level and motivation of the student. Depending on the level, a flag (red, orange or green) is generated to orientate the student towards the best path and with the best workload according to the student's profile (Fig. 6). A typical scenario consists of: (i) videopresentation of the lab; (ii) test on prerequisites; (iii) lesson (online documents); (iv) formative test; (v) remote labworks with three levels of difficulty; (vi) comparison with simulator (virtual lab); (vii) final summative test with upload of work report; (viii) satisfaction survey. Fig. 7 is a snapshot of the screen seen by a client when asking for the remote lab within the LMS: he can download a preformatted text-file for writing the report to answer the questions (left button), click on "Travaux Pratiques" (middle button) to run real experiment, or run the simulator (right button). The traffic light indicates the best difficulty level to choose from, depending on previous answers to quizzes. The student can also watch a tutorial that explains the use of the remote lab by playing a video ${ }^{1}$ (bottom movie).

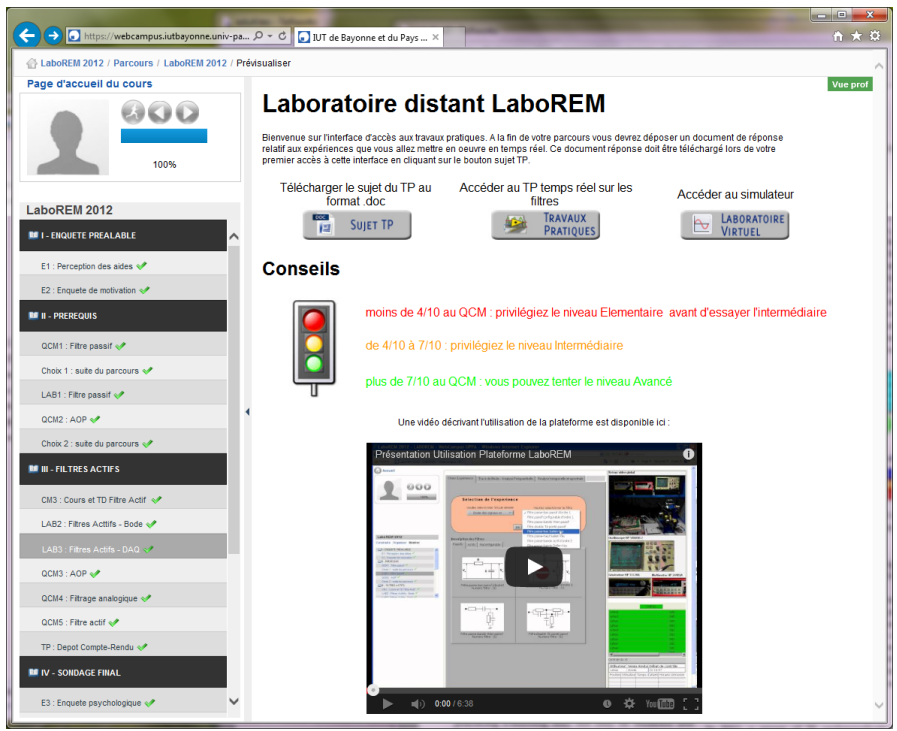

Figure 7. LMS interface seen by the client when requesting access to remote lab experiment.

\footnotetext{
${ }^{1}$ https://www youtube. com/watch?feature=player_ embedded\&v $=\mathrm{m} 7$ py Ir $2 u b 54$
} 
Table 1. Student motivation (on a 5-point Likert scale)

\begin{tabular}{|c|c|c|c|c|}
\hline year (\# students) & \multicolumn{2}{|c|}{ Intrinsic } & \multicolumn{2}{|c|}{ Extrinsic } \\
\hline Pre-Inquiry & mean & increment & mean & increment \\
\hline 2011 (29 stud.) & 3.73 & & 2.06 & \\
\hline 2012 (22 stud.) & 3.89 & & 1.95 & \\
\hline 2013 (25 stud.) & 4.18 & +0.29 & 2.42 & +0.47 \\
\hline Post-Inquiry & mean & increment & mean & increment \\
\hline 2012 (22 stud.) & 3.1 & & 2.55 & \\
\hline 2013 (25 stud.) & 3.5 & +0.4 & 3.1 & +0.55 \\
\hline
\end{tabular}

\subsection{Student Motivation}

In 2011, a group of 29 students was tested. At first, an a priori survey (Pre-Inquiry) about their motivation $^{2}$ showed that their main motivation was intrinsic acquisition of knowlegde (mean score of about 3.8/5), long before their position with respect to others (mean score of about 2/5). To enhance their external motivation, a Top 10 list was added in 2013, that gives in real-time the best score achieved in measurement activity ("hall of fame"). Moreover, the robotic arm was also added. This proved to increase extrinsic motivation by more than $20 \%$. Tab. 1 gives the average values for intrinsic and extrinsic motivation measured from pre- and post-inquiries ${ }^{3}$ (before and after the lab activity) in 2011, 2012 and 2013. It also shows (in bold) the increments between 2012 and 2013. Worth to mention is the fact that the students that most participated in the Top 10 were not necessarily the "best" of our students (in terms of academic marks). Indeed, competitiveness increased their motivation.

\section{DISCUSSION AND FUTURE WORK}

If motivation increase is proven, we do not claim yet better learning outcomes, compared to hands-on. The LaboREM platform is one supplementary tool to help students in their studies, especially in an autonomous and student-centered context, as recommended in Europe by the Bologna process. Moreover, this tool is exhibited each year during the "open doors day" of the university and proves to be very attractive for visitors which are teenagers coming from high schools. It contributes to increase the number of students willing to undergo a STEM curriculum (science, technology, engineering, mathematics). The perspective of this work is manifold. A multi-server solution is of high interest to give access to experiments in various remote labs (cf. iLab shared architecture). We intend to make LaboREM fully compliant with SCORM standard for complete integration of the weblab into the

\footnotetext{
${ }^{2}$ Sample statements for measuring motivation are, among others: "It is important for me to learn"; "It is important for me to look like being proficient"; "I am motivated by this remote learning activity"; "I am motivated by the final mark I will obtain for this labwork".

${ }^{3}$ Pre- and Post-inquiry values are not directly comparable since they are based on two different types of questionnaire; moreover, in 2011, there was no Post-inquiry available.
}

LMS. As regards image processing, face expression analysis may help to know the state of motivation of a student. Giving control of the camera to the client is attractive for closer collaboration with a colleague or tutor. In addition to the wide-angle camera that supervises the whole scene, a minidrone equipped with a micro-camera can be used to fly over the lab at user's request. Other labwork to be added is to pilot photovoltaic panels on the roof of the university buildings.

\section{REFERENCES}

Dutta, S., Prakash S., Estrada D., \& Pop E. (2011, November). A web-service and interface for remote electronic device characterization. IEEE Trans. on Education 54(4), 646-651.

Garcia-Zubia, J. \& Alves G. R. (Eds.) (2011). Using Remote Labs in Education: two little ducks in remote experimentation. Bilbao, Spain: University of Deusto. ISBN 978-84-9830-398-8.

Garcia-Zubia, J., Orduña P., Lopez-de-Ipiña D., \& Alves G. R. (2009, December). Addressing software impact in the design of remote laboratories. IEEE Trans. on Industrial Electronics 56(12), 4757-4766.

Guimaraes, E. G., Cardozo E., Moraes H., \& Coelho P. R. (2011, April-June). Design and implementation issues for modern remote laboratories. IEEE Trans. on Learning Technologies 4(2), 149-161.

Harward, V. J., del Alamo J. A., Lerman S. R., Bailey P. H., $\&$ al. (2008, June). The iLab Shared Architecture: A web services infrastructure to build communities of internet accessible laboratories. Proc. of the IEEE 96(6), 931-950.

Luthon, F., Petre A., Steriu D., \& Besleaga A. (2009, Nov.). LaboRem: open lab for remote work. In $3 r d$ Int. Conf. on Signals, Circuits and Systems (SCS'09), Jerba, Tunisia, pp. 1-6. IEEE.

Mendez, J. A. \& Gonzalez E. J. (2011, November). Implementing motivational features in reactive blended learning: Application to an introductory control engineering course. IEEE Trans. on Education 54(4), 619-627.

Nedic, Z. \& Machotka J. (2007). Remote laboratory Net$\mathrm{Lab}$ for effective teaching of 1 st year engineering students. Int. Journal of Online Engineering iJOE 3(3).

Orduña, P., Uribe S. B., Isaza N. H., Sancristobal E., Emaldi M., Martin A. P., DeLong K., Bailey P., Ipiña de D. L., Castro M., \& Garcia-Zubia J. (2013). Generic integration of remote laboratories in learning and content management systems through federation protocols. In 43rd Annual Frontiers in Education Conf. (FiE 2013), Oklahoma, US, pp. 1372-1378. IEEE.

Steriu, M. \& Luthon F. (2006, Sep. 24-27). Open architecture for signal processing lab distance learning. In 4th IEEE Digital Signal Processing Education Workshop (DSP'06), Jackson Lake Lodge, Wyoming, USA, pp. 305-310.

Tawfik, M., Sancristobal E., Martin S., Gil R., Diaz G., Peire J., Castro M., Nilsson K., Zackrisson J., Håkansson L., \& Gustavsson I. (2013, January). Virtual instrument systems in reality (VISIR) for remote wiring and measurement of electronic circuits on breadboard. IEEE Trans. on Learning Technologies 6(1), 60-72. 